The Journal of Toxicological Sciences, Vol. 16, 181-190, 1991

\title{
ADVANTAGES OF TRIPLE THERAPY WITH MIZORIBINE, CYCLOSPORINE AND PREDNISOLONE OVER OTHER TYPES OF TRIPLE AND/OR DOUBLE THERAPY INCLUDING CYCLOSPORINE FOR RENAL TRANSPLANTATION
}

\author{
Hisanori UCHIDA, Kunshi MITA, Yoshibumi BEKKU, \\ Youji NISHIMURA, Yuichi ISHIDA, Kenji WATANABE, \\ Shinji TOMIKAWA, Sumio INOUE, Hisayuki SUGIMOTO, \\ Takeshi NAGAO, Tsuneo OSAKABE* ${ }^{*}$, Yoshisuke NAKAYAMA*, \\ Kazuo YOKOTA*, Koushi SATO*, Tadao ENDO** \\ and Mitsuhito OKUBO*** \\ Departments of Surgery and Organ Transplantation, \\ Institute of Medical Science, University of Tokyo, \\ 4-6-1 Shiroganedai, Minato-ku, Tokyo 108, Japan \\ Departments of ${ }^{*}$ Surgery, ${ }^{* *}$ Urology and ${ }^{* * *}$ Internal Medicine, \\ Kitasato University, 15-1 Kitasato 1 chome, Sagamihara, Kanagawa 228, Japan
}

Accepted November 1, 1991

\begin{abstract}
Mizoribine $(\mathrm{Mz})$ is an analogue of azathioprine $(\mathrm{Az})$ with less hepatotoxicity, being extensively used as immunosuppressant in place of the latter agent especially in Japan. However, careful comparative studies of mizoribine $(\mathrm{Mz})$, cyclosporine $(\mathrm{Cy})$, and prednisolone $(\operatorname{Pr})$ versus azathioprine $(\mathrm{Az}), \mathrm{Cy}$ and $\operatorname{Pr}$ or $\mathrm{Cy}$ and $\operatorname{Pr}$ in renal allotranspalnt patients have not been reported. Retrospectively we compared triple therapy with $\mathrm{Mz}, \mathrm{Cy}$, and $\operatorname{Pr}$ (group I, $\mathrm{n}=50$ ) to triple therapy with $\mathrm{Az}, \mathrm{Cy}$ and $\operatorname{Pr}$ (group II, $n=13$ ) and/or double therapy with $C y$ and $\operatorname{Pr}$ (group III, $n=11$ ) in one-haplotype-identical living related renal transplantations performed between Oct. 1984 through March 1989. Initial and maintenance doses of Cy in groups I and II were largely two thirds of those in group III. Patient and graft survival rates at 3 years in each group are $100 \%$ and $92 \%$ (group I), $100 \%$ and $91 \%$ (group II), and $91 \%$ and $82 \%$ (group III). There were no statistical differences in patient and graft survival rates between these three groups. The incidences of miscellaneous complications were the same in the groups. Bone marrow suppression, however, was significantly less in group I than in group II $(\mathrm{P}<0.005)$. Cy related nephrotoxicity was apparently less in groups I and II than in group III. Estimated US $\$ 5,000$ in a year can be saved by immunosuppressive treatment in a patient of group I as
\end{abstract}

Correspondence : Dr. Hisanori UCHIDA at the above address.

Supported in part by Research Grant C63570607 from the Ministry of Education 
H. UCHIDA et al.

compared to a patient in group III. Therefore, we conclude that triple therapy with $\mathrm{Mz}, \mathrm{Cy}$ and $\operatorname{Pr}$ is superior to those with $\mathrm{Az}, \mathrm{Cy}$ and $\mathrm{Pr}$, and/or double therapy with $\mathrm{Cy}$ and $\operatorname{Pr}$.

Key words: One-haplotype-identical renal allotransplant, living related donor, mizoribine, cyclosporine, azathioprine, life survival rate, graft survival rate, complication, bone marrow suppression.

\section{INTRODUCTION}

Since the introduction of cyclosporine (Cy) to clinical renal allotransplantation, remarkable progress in the graft survival rate has been achieved (Kahan, 1985 ; Starzl et al., 1980). Several problems related to the use of $\mathrm{Cy}$ in clinical patients, however, have also been reported. Nephrotoxicity is the most troubling adverse reaction of this agent (Kahan, 1985 ; Mihatsch et al., 1985). Triple drug immunosuppression with $\mathrm{Cy}$, azathioprine $(\mathrm{Az})$ and steroids $(\mathrm{St})$ is one modality for reducing dosage and avoiding the untoward effects of Cy (Canafax et al., 1985 ; Lorber et al., 1985). However, bone marrow suppression and hepatotoxicity caused by $\mathrm{Az}$ can be an another potential hazard in this triple drug immunosuppression. Mizoribine $(\mathrm{Mz})$, developed in the laboratory of Toyo-Jozo Co. (Mizuno et al., 1974) and applied to clinical renal allotransplantation in 1980 (Inou et al., 1981; Uchida et al., 1982), is an alternative to $\mathrm{Az}$ that carries less danger of bone marrow suppression and/or hepatotoxicity (Aso et al., 1987).

In this clinical study triple drug immunosuppression with $\mathrm{Mz}, \mathrm{Cy}$ and $\mathrm{St}$ was compared with double drug immunosuppression with $\mathrm{Cy}$ and $\mathrm{St}$, and triple drug immunosuppression with $\mathrm{Az}, \mathrm{Cy}$ and St.

\section{MATERIALS AND METHODS}

In two consecutive series of one-haplotype-identical living related renal allotransplant recipients treated since 1984 in two different institutions, Kitasato University and the University of Tokyo, prospective recipients were allocated randomly, and intentionally in part, to three groups of immunosuppressive therapy. Group I comprised 48 recipients treated with $\mathrm{Mz}, \mathrm{Cy}$ and St. The initial and maintenance doses of $\mathrm{Mz}$ were $2 \mathrm{mg} / \mathrm{kg} / \mathrm{day}$. The dose of Cy was $6 \sim 10 \mathrm{mg} / \mathrm{kg} /$ day initially and $2 \sim 4 \mathrm{mg} / \mathrm{kg} / \mathrm{day}$ during maintenance. The dose of St was $1 \sim 1.4$ $\mathrm{mg} / \mathrm{kg} /$ day initially. This was tapered down by $0.2 \mathrm{mg} / \mathrm{kg} / \mathrm{day}$ every week to a maintenance dose of $0.2 \mathrm{mg} / \mathrm{kg} /$ day $2 \sim 3$ months later. Group II comprised 13 patients treated with $\mathrm{Az}, \mathrm{Cy}$ and St. The initial and maintenance doses of $\mathrm{Az}$ were 1 $\mathrm{mg} / \mathrm{kg} / \mathrm{day}$. The initial and maintenance doses of $\mathrm{Cy}$ and $\mathrm{St}$ were the same as those in group I. Potential recipients in group II who showed hepatic functional abnormalities indicated by high S-GOT and/or S-GPT ( $\geqq 50 \mathrm{IU} / \mathrm{L}$ ) levels or 
demonstrated a small number of peripheral WBC $(\leqq 4000 / \mathrm{cmm})$ at transplant surgery were allocated to group I. Group III comprised 11 patients who treated by double drug immunosuppression with $\mathrm{Cy}$ and St. The initial and maintenance doses of Cy in this group of recipients were $10 \sim 14 \mathrm{mg} / \mathrm{kg} /$ day and $4 \sim 6 \mathrm{mg} / \mathrm{kg} /$ day, respectively. The dose schedule for St was the same as in groups I and II.

In each group of patients the original immunosuppressive dose schedules were occasionally changed because of various complications. After the complications had treated, care was taken to return to the original dose schedule.

Rejection crisis was treated by two to four administrations of pulse therapy consisting of methylprednisolone $(10 \mathrm{mg} / \mathrm{kg})$, supplemented at times by local irradiation.

There were no statistically significant differences in recipient age and sex, donor age and sex, or donor-recipient relationships between the groups. There were also no statistically significant differences in the number of HLA-A, -B and -DR antigen matches and mismatches.

Patients and graft survival rates were calculated by actuarial techniques. Graft loss was defined as either return to dialysis or death with a functioning graft. All causes of graft loss were included, as well as all deaths.

The incidence of rejection episodes that occurred during the initial 3 months was calculated and comparisons were made between the groups. Miscellaneous complications arising within 3 months after transplantation were also collected and compared.

Hepatic dysfunction was defined as the development of high S-GPT or S-GOT levels $(\geqq 100 \mathrm{IU} / \mathrm{L})$ in at least one set of repeated blood collections. Bone marrow suppression was defined as a decrease in the number of peripheral WBC to less than $3000 / \mathrm{cmm}$. Patients with immunosuppression-induced diabetes were defined as those who needed insulin therapy during the postoperative course. Patients who needed insulin therapy before transplantation were not included in this study.

\section{RESULTS}

\section{Acutuarial Patient and Graft Survival Rates :}

Actuarial patient and graft survival rates are summarized in Figs. 1 and 2. In groups I and II no patient loss was experienced during the observation period. On the other hand, in group III, one patient died 3 months after transplantation of acute pancreatitis combined with Pneumocystis carinii pneumonia but with a functioning allograft. Therefore, the actuarial patient survival rate for group III was $91 \%$ at 3 months and remained stable thereafter. None of the differences in patient survival rates between groups I, II and III was statistically significant. The acutuarial graft survival rate in group I was $97.9 \%$ at one year, decreasing slightly thereafter. The graft survival rates in groups II and III were 90.9 and $81.8 \%$, respectively, at one year and remained stable at 3 years. None of the differences in graft survival rates 
H. UCHIDA et al.

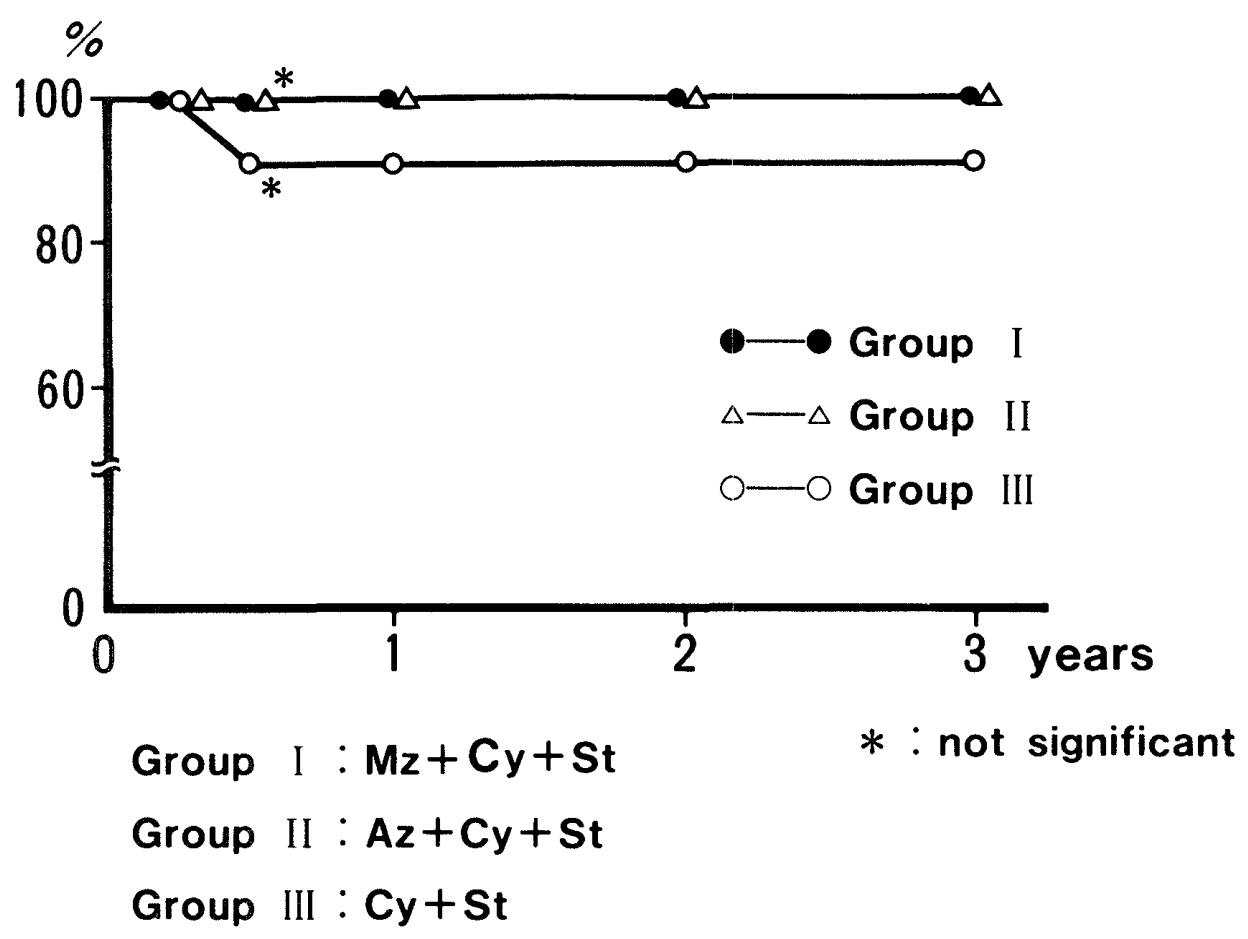

Fig. 1. Actuarial life survival rate of one-haplotype-identical living related renal allotransplant recipients treated by three different modes of immunosuppression. Patients in group 1 were treated by mizoribine $(\mathrm{Mz})$, cyclosporine $(\mathrm{Cy})$ and steroids $(\mathrm{St})$. Patients in group 2 were treated by azathioprine $(\mathrm{Az})$, Cy and St. Patients in group 3 were treated by $\mathrm{Cy}$ and $\mathrm{St}$. There were no significant differences in patient survival rates.

between groups I, II and III was statistically significant.

Comparison of Incidence of Rejection Episodes :

Acute rejection crisis occurred in 18 of 48 recipients (37.5\%) within 3 months after transplantation in group I. Likewise in groups II and III, episodes of acute rejection crisis were observed in 4 of $13(30.8 \%)$ and 6 of 11 recipients $(54.5 \%)$, respectively (Table 1). The incidence of acute rejection episodes was not significantly different between groups I, II and III.

\section{Comparison of Renal Function:}

Renal function, expressed as the level of serum creatinine, in each group is summarized in Fig. 3. A gradual increase in serum creatinine level occurred in each group during 3 years after transplantation. In groups I and II levels of serum creatinine were significantly lower than that in group III within 6 months after transplantation. Between one year and 3 years after transplantation, there were no statistically significant differences in serum creatinine levels of between groups I, II and III. 
Advantages of triple therapy

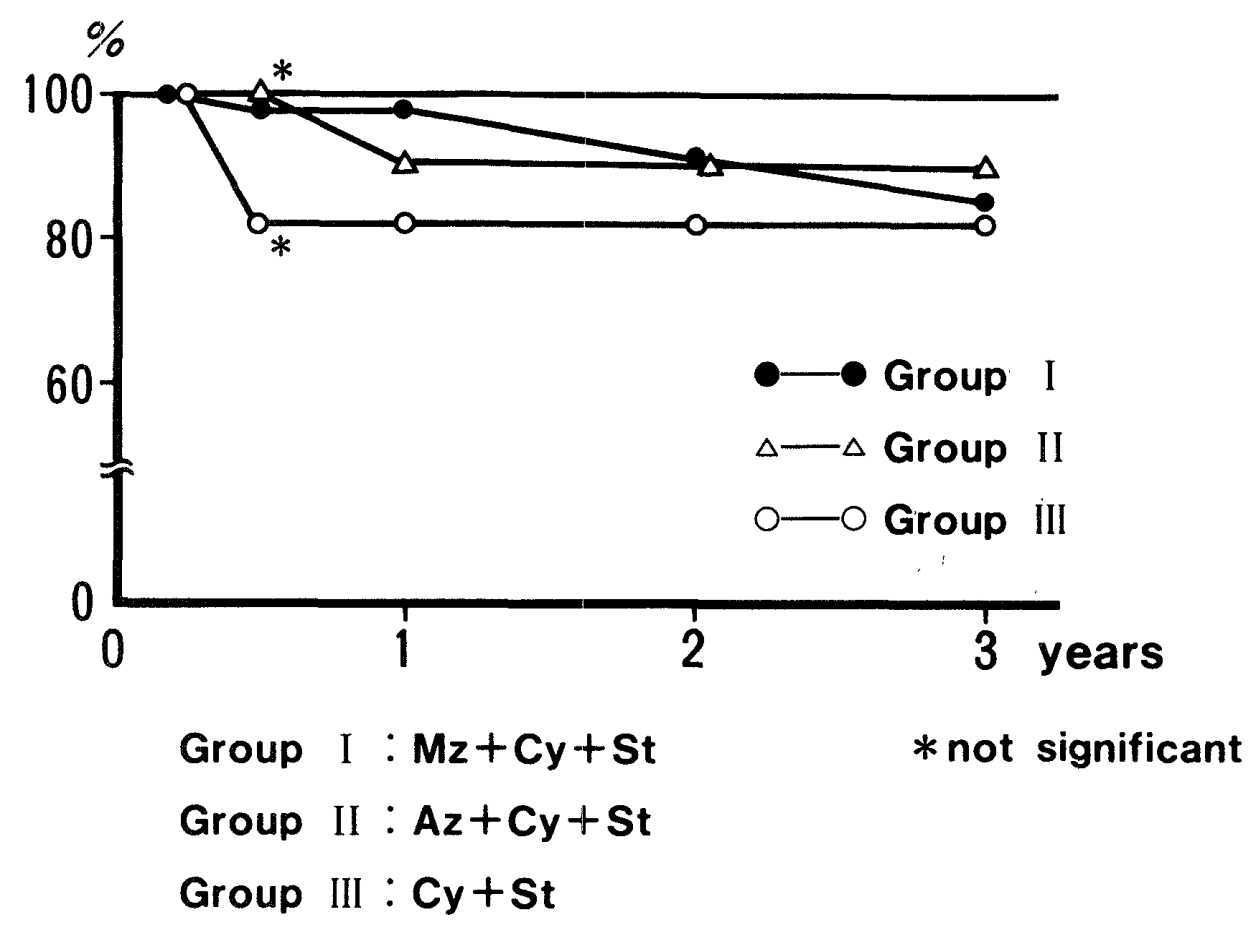

Fig. 2. Actuarial graft survival rate of one-haplotype-identical living related renal allotransplant recipients treated by three different modes of immunosuppression. Patients in group 1 were treated by mizoribine $(\mathrm{Mz})$, cyclosporine $(\mathrm{Cy})$ and steroids (St). Patients in group 2 were treated by azathioprine (Az), Cy and St. Patients in group 3 were treated by $\mathrm{Cy}$ and $\mathrm{St}$. There were no significant differences in graft survival rates.

Table 1. Incidence of rejection episodes within 3 months.

\begin{tabular}{lllll}
\hline \hline & & Group I & Group II & Group III \\
\hline No. of recipients & & 48 & 13 & 11 \\
Rejection episodes & Yes & $18(37.5 \%)^{*}$ & $4(30.8 \%)^{* *}$ & $6(54.5 \%)^{* * *}$ \\
& No & $30(62.5 \%)$ & $9(69.2 \%)$ & $5(45.5 \%)$ \\
\hline
\end{tabular}

Group I: Mz, Cy and St

Group II: $\mathrm{Az}, \mathrm{Cy}$ and $\mathrm{St}$

Group III: Cy and $\mathrm{St}$

* versus $^{* *}$ : not significant

* versus ${ }^{* * *}$ : not significant

${ }^{* *}$ versus $^{* * *}$ : not significant 
H. UCHIDA et al.

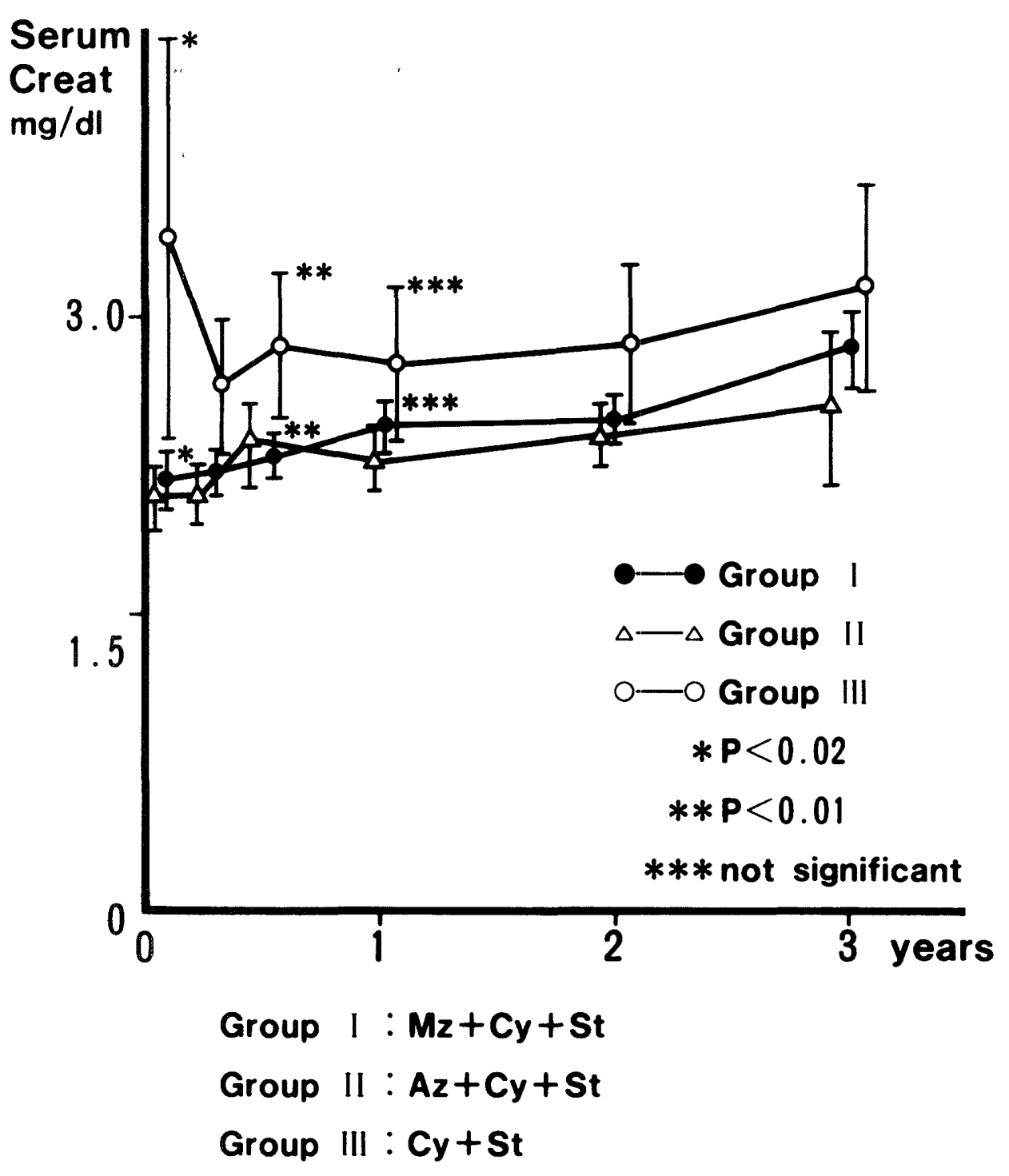

Fig. 3. Levels of serum creatinine in three different groups of immunosuppression. Levels of serum creatinine in group 1 are significantly lower than those in group 3 within one year after transplantation.

$\mathrm{Mz}$ : mizoribine, $\mathrm{Cy}$ : cyclosporine, $\mathrm{Az}$ : azathioprine, $\mathrm{St}$ : steroids

Comparison of Incidence of Miscellaneous Complications Due to Immunosuppression within 3 Months :

Bone marrow suppression occurred in 3 of 48 recipients $(6.3 \%)$ in group I and 5 of 13 recipients $(38.5 \%)$ in group II, a significant difference $(P<0.005)$. The incidence of bone marrow suppression was also significantly lower in group III than group II $(\mathrm{P}<0.05)$ (Table 2$)$.

Grave systemic infections such as bacterial, fungal, protozoal and viral lung or central nervous system infections occurred in 5 of 48 recipients $(10.4 \%)$ in group I and 5 of 13 recipients $(38.5 \%)$ in group II. The difference was statistically 
Advantages of triple therapy

Table 2. Incidence of miscellaneous complications due to immunosuppression within 3 months.

\begin{tabular}{|c|c|c|c|}
\hline & Group I & Group II & Group III \\
\hline No. of recipients & 48 & 13 & 11 \\
\hline Bone marrow suppression & $3(6.3 \%)^{*}$ & $5(38.5 \%)^{* *}$ & $0 \quad(0 \%)^{* * *}$ \\
\hline Grave systemic infections & $5(10.4 \%)^{+}$ & $5(38.5 \%)^{++}$ & $2 \quad(18.2 \%)$ \\
\hline Hepatic dysfunction & $3(6.3 \%)^{\#}$ & $2 \quad(18.2 \%)$ & $6 \quad(54.5 \%)^{\# \#}$ \\
\hline $\begin{array}{l}\text { Immunosupprresion-induced } \\
\text { diabetes }\end{array}$ & $2(4.2 \%)$ & $1(7.7 \%)$ & $0 \quad(0 \%)$ \\
\hline
\end{tabular}

Group I : $\mathrm{Mz}, \mathrm{Cy}$ and $\mathrm{St}$

Group II : $\mathrm{Az}, \mathrm{Cy}$ and $\mathrm{St}$

Group III : $\mathrm{Cy}$ and $\mathrm{St}$

* versus ** $: \mathrm{P}<0.005$ significant

** versus ${ }^{* * *}: \mathrm{P}<0.05$ significant

+ versus $^{++}: \mathrm{P}<0.02$ significant

\# versus \#\# $: \mathrm{P}<0.005$ significant

significant $(\mathrm{P}<0.02)$ (Table 2).

There were no significant differences in the incidence of hepatic dysfunction or immunosuppression-induced diabetes in groups I and II.

\section{DISCUSSION}

Clinical experiences of triple therapy with $\mathrm{Mz}, \mathrm{Cy}$ and $\mathrm{St}$ in renal allotransplantation have been reported from a number of transplant centers in Japan including ours (Amemiya et al., 1989; Aso et al., 1987; Takahara et al., 1988). Patient and graft survival rates, so far, have been comparable to those of triple therapy with $\mathrm{Az}, \mathrm{Cy}$ and $\mathrm{St}$ reported by many investigators. However, a prospective randomized comparative study of both triple therapies has not been reported. Therefore, whether triple therapy with $\mathrm{Mz}, \mathrm{Cy}$ and $\mathrm{St}$ is better than that with $\mathrm{Az}, \mathrm{Cy}$ and St has not been determined, although treatment with $\mathrm{Mz}$ instead of $\mathrm{Az}$ has theoretical advantages, since $\mathrm{Mz}$ has less bone marrow suppression and hepatotoxicity than Az. In the present study, recipients treated with $\mathrm{Az}, \mathrm{Cy}$ and $\mathrm{St}$ and having normal peripheral WBC were intentionally selected by excluding patients with potential suppression of bone marrow. Still, $38.4 \%$ of the recipients had developed a decrease in peripheral WBC to less than $3000 / \mathrm{cmm}$ by 3 months after transplantation. In contrast, only $6.3 \%$ of recipients treated with $\mathrm{Mz}, \mathrm{Cy}$ and $\mathrm{St}$ developed bone marrow suppression. This difference was statistically significant. Moreover, systemic infections were more frequently encountered in recipients treated with $\mathrm{Az}, \mathrm{Cy}$ and St than those treated with $\mathrm{Mz}, \mathrm{Cy}$ and St. Likewise, recipients treated with $\mathrm{Az}, \mathrm{Cy}$ and $\mathrm{St}$ were intentionally selected in part to have normal hepatic function at transplantation. 


\section{H. UCHIDA et al.}

Patients with abnormal levels of S-GOT or S-GPT were grouped to immunosuppression with $\mathrm{Mz}, \mathrm{Cy}$ and $\mathrm{St}$. With respect to postoperative hepatic dysfunction, however, $15.4 \%$ of the former group of patients developed high S-GOT and/or S-GPT levels, whereas only $6.3 \%$ of the latter group of patients developed abnormal hepatic functions. However, the difference was not statistically significant.

$\mathrm{Mz}$ is an imidazol nucleoside isolated from the culture filtrate of a true fungus, Eupenicillium brefeldianum, and is known to suppress cell-mediated and humoral immunity by inhibiting conversion from inosinic acid monophosphate (IMP) to guanylic acid monophosphate (GMP) during nucleic acid synthesis (Sakaguchi et al., 1975). In comparison studies of $\mathrm{Mz}$ with $\mathrm{Az}$ based on large animal experiments done in our laboratory and others, $\mathrm{Mz}$ appeared to be less hepatotoxic and less suppressive to bone marrow than Az (Sagawa et al., 1978; Yokota, 1980). In long-term toxicological studies on Beagle dogs, Uchida and his associates found no blood or biochemical abnormalities, or morphological changes in various tissues and organs including the liver and bone marrow (Uchida et al., 1985). The results of the present study confirmed those of previous reports.

The dose of $\mathrm{Cy}$ in double drug treatment with $\mathrm{St}$ in this study was 1.4 times more than the initial and 1.6 times more than the maintenance dose in triple drug treatment. Nephrotoxicity due to $\mathrm{Cy}$ in both triple drug therapies was apparently less significant, as indicated by serum creatinine levels. Osakabe and his associates found that combined therapy with $\mathrm{Cy}$ and $\mathrm{Mz}$ prolonged the survival of canine renal allografts, demonstrating a synergistic effect of the two agents (Osakabe et al., 1989).

The rationale for triple therapy consisting of $\mathrm{Mz}, \mathrm{Cy}$ and St with $\mathrm{Mz}$ in place of $\mathrm{Az}$ or instead of double therapy with $\mathrm{Cy}$ and St appeared to have been confirmed in the present study, although a randomized prospective study is needed.

In addition, treatment with $\mathrm{Mz}, \mathrm{Cy}$ and St can save an estimated US $\$ 5,000$ in medical costs per year compared with treatment with $\mathrm{Cy}$ and St. This would represent a considerable saving to patients with economic considerations. From this point as well, triple therapy with $\mathrm{Mz}$, Cy and St is advisable.

\section{CONCLUSION}

Immunosuppression by $\mathrm{Mz}, \mathrm{Cy}$ and $\mathrm{St}$ in one-haplotype-identical living related renal allotransplant recipients demonstrated the same life and graft survival rates as that by Az, Cy and St or Cy and St. Treatment with Mz, Cy and St resulted in significantly less bone marrow suppression and grave systemic infection compared with $\mathrm{Az}, \mathrm{Cy}$ and St treatment. In the group of patients treated with $\mathrm{Mz}, \mathrm{Cy}$ and $\mathrm{St}$, the level of serum creatinine was significantly lower than that in group treated with $\mathrm{Cy}$ and St. Therefore, immunosuppressive treatment with $\mathrm{Mz}, \mathrm{Cy}$ and $\mathrm{St}$ is better than that with Az, Cy and St or Cy and St. 


\section{REFERENCES}

Amemiya, H., Suzuki, S., Watanabe, H., et al. (1989) : Synergistically enhanced immunosuppressive effect by combined use of cyclosporine and mizoribine. Transplant. Proc., 21, 956-958.

Aso, K., Uchida, H., Sato, K., et al. (1987) : Immunosuppression with low-dose cyclosporine combined with Bredinin and cyclosporine. Transplant. Proc., 19, 1955-1958.

Canafax, D. M., Martel, E. J., Ascher, N. L., et al. (1985) : Two methods of managing cyclosporine nephrotoxicity: Conversion to azathioprine, prednisolone or cyclosporine, azathioprine, and prednisolone. Transplant. Proc., 17 (Supple. 1), 282-285.

Inou, T., Kusaba, R., Takahashi, I., et al. (1981) : Clinical trial of Bredinin in renal transplantation. Transplant. Proc., 13, 315-318.

Kahan, B. D. (1985) : Cyclosporine: The agent and its actions. Transplant. Proc., 17 (Supple. 1), 5-18.

Lorber, M. I., Flechner, S. M., Van Buren, C. T., et al. (1985) : Cyclosporine, azathioprine, and prednisolone as treatment for cyclosporine-induced nephrotoxicity in renal transplant recipients. Transplant. Proc., 17 (Supple. 1), 282-285.

Mihatsch, M. J., Thiel, G., Basler, V., et al. (1985) : Morphological patterns in cyclosporine-treated renal transplant recipients. Transplant. Proc., 17(Supple. 1), 101-116.

Mizuno, K., Tsujino, M., Takada, M., et al. (1974): Studies on Bredinin I. Isolation, characterization and biological properties. J. Antibiot., (Tokyo) 27, 775-782.

Osakabe, T., Uchida, H., Masaki, Y., et al. (1989) : Studies on immunosuppression with low dose cyclosporine combined with mizoribine in experimental and clinical cadaveric renal allotransplantation. Transplant. Proc., 21, 1598-1600.

Sagawa, S., Ishibashi, M., Arima, M., et al. (1978) : Studies on development of new immunosuppressive agent-Immunosuppressive effectiveness of Bredinin on canine kidney alltransplant survival-. Ishoku, 13, 271-276.

Sakaguchi, K., Tsujino, M., Yoshizawa, M., et al. (1975) : Action of Bredinin on mammalian cells. Cancer Res., 35, 1643-1648.

Starzl, T. E., Weil, R. III, Iwatsuki, S., et al. (1980): The use of cycloporin A and prednisolone in cadaver kidney transplantation. Surg. Gynec. Obstet., 151, 17-26.

Takahara, S., Fukunishi, T., Kokado, Y., et al. (1988) : Combined immunosuppression with low-dose cyclosporine, mizoribine, and prednisolone. Transplant. Proc., 20 (Supple. 3), 147-151.

Uchida, H., Masaki, Y., Taira, M., et al. (1985) : Long-term toxicological studies with mizoribine $\left(\right.$ Bredinin $^{\mathrm{R}}$ ) in beagle dogs (The first report). J. Toxicol. Sci., 


\section{H. UCHIDA et al.}

$10,35-49$.

Uchida, H., Yokota, K., Osakabe, T., et al. (1982): A comparative study of tripple-drug treatment with mizoribine, azathioprine, steroids versus doubledrug treatment with azathioprine and steroids in clinical renal allotransplantation. Ishoku, 17 (Supple.), 691-700.

Yokota, K. (1980): Effect of mizoribine on canine kidney allograft survival in comparison with the conventional immunosuppressive therapy. Nippon Geka Gakkai Zasshi, 83, 769-782. 